\title{
Effectiveness of Sodium Picosulfate/Magnesium Citrate (PICO) for Colonoscopy Preparation
}

\author{
Ki Hwan Song, Wu Seok Suh, Jin Sik Jeong, Dong Sik Kim, Sang Woo Kim, Dong Min Kwak, \\ Jong Seong Hwang, Hyun Jin Kim, Man Woo Park, Min Chul Shim, Ja-Il Koo, Jae Hwang Kim ${ }^{1}$, Dae Ho Shon ${ }^{2}$ \\ Coloproctology Center, Goo Hospital, Daegu; ${ }^{1}$ Department of Surgery, Yeungnam University School of Medicine, Daegu; ${ }^{2}$ Hwanggeumbit \\ Hakmoon Colorectal Surgery Clinic, Daegu, Korea
}

Purpose: Bowel preparation with sodium phosphate was recently prohibited by the U.S. Food and Drug Administration. Polyethylene glycol (PEG) is safe and effective; however, it is difficult to drink. To identify an easy bowel preparation method for colonoscopy, we evaluated three different bowel preparation regimens regarding their efficacy and patient satisfaction. Methods: In this randomized, comparative study, 892 patients who visited a secondary referral hospital for a colonoscopy between November 2012 and February 2013 were enrolled. Three regimens were evaluated: three packets of sodium picosulfate/magnesium citrate (PICO, group A), two packets of PICO with 1 L of PEG (PICO + PEG 1 L, group B), and two packets of PICO with $2 \mathrm{~L}$ of PEG (PICO + PEG $2 \mathrm{~L}$, group C). A questionnaire survey regarding the patients' preference for the bowel preparation regimen and satisfaction was conducted before the colonoscopies. The quality of bowel cleansing was scored by the colonoscopists who used the Aronchick scoring scale and the Ottawa scale.

Results: The patients' satisfaction rate regarding the regimens were $72 \%$ in group A, $64 \%$ in group B, and $45.9 \%$ in group C. Nausea and abdominal bloating caused by the regimens were more frequent in group $\mathrm{C}$ than in group $\mathrm{A}$ or group $\mathrm{B}(\mathrm{P}<$ $0.01)$. Group $C$ showed the lowest preference rate compared to the other groups $(\mathrm{P}<0.01)$. Group $\mathrm{C}$ showed better right colon cleansing efficacy than group A or group B.

Conclusion: Group A exhibited a better result than group B or group C in patient satisfaction and preference. In the cleansing quality, no difference was noted between groups A and C.

Keyword: Sodium picosulfate magnesium citrate; Satisfaction; Bowel preparation; Polyethylene glycols; Colonoscopy

\section{INTRODUCTION}

Adequate bowel preparation is essential for an accurate colonoscopic examination. Polyethylene glycol (PEG) and sodium phosphate $(\mathrm{NaP})$ have been commonly used for bowel preparation before colonoscopy or colorectal surgery [1]. Recently, the U.S. Food and Drug Administration (FDA) prohibited the use of $\mathrm{NaP}$ for

Received: August 16, 2013 - Accepted: May 21, 2014

Correspondence to: Ki Hwan Song, M.D.

Coloproctology Center, Goo Hospital, 14 Gamsambuk-gil, Dalseo-gu,

Daegu 704-954, Korea

Tel: +82-53-560-9120, Fax: +82-53-558-1163

E-mail: song3677@chol.net

(C) 2014 The Korean Society of Coloproctology

This is an open-access article distributed under the terms of the Creative Commons Attribution NonCommercial License (http://creativecommons.org/licenses/by-nc/3.0) which permits unrestricted noncommercial use, distribution, and reproduction in any medium, provided the original work is properly cited. bowel preparation because of renal failure.

Picolight (PICO) has an acceptable taste and patient tolerance because of the small volume required, and its bowel preparation efficacy is comparable to that of the PEG solution [2]. The primary elements of PICO are sodium picosulfate, magnesium oxide, and citric acid. These elements have a combined effect that stimulates peristalsis and induces a shift of water into the lumen [2]. In solution, magnesium oxide and citric acid combine to form magnesium citrate, which acts as an osmotic laxative, whereas sodium picosulfate acts as a stimulant laxative. PICO is used in relatively small volumes (each packet is mixed with $150 \mathrm{~mL}$ of water) and has a more pleasant taste than PEG [1].

In previous studies on PICO, PEG, and NaP, PICO, and PEG showed similar results in bowel cleansing; however, PICO was better than PEG and comparable to $\mathrm{NaP}$ in terms of adverse events. Pico has been used for two decades in the European Union, the United Kingdom and Canada [2-4]. However, few clinical studies 
have been published in Korea, and no studies have reported on a combined regimen of PICO with PEG. We compared three different regimens to discover an easy and effective bowel preparation method for colonoscopy. PICO in three packets (group A), PICO in two packets with $1 \mathrm{~L}$ of PEG (group B) and PICO in two packets with 2 L of PEG (group C) were evaluated according to their cleansing efficacy, patient satisfaction, and patient preference.

\section{METHODS}

\section{Patients}

From November 2012 to February 2013, 892 healthy, volunteer patients, aged from 20 to 70 years, were enrolled in this study in our hospital's coloproctology center. We obtained informed consent for the bowel preparation method before colonoscopy. Each patient was randomly assigned into one of the three groups according to the scheduled colonoscopy time. Each patient and colonoscopist was blind to which bowel preparation drugs were used. The patients with the following characteristics were excluded from the study: an inability to give informed consent, heart failure, renal dysfunction, liver disease, metabolic disease, previous colorectal surgery, and previous hypersensitivity to bowel preparation.

\section{Bowel preparation method}

Each colonoscopy was scheduled at $9 \mathrm{AM}$, and all the patients had a meal at 6 PM the day before the colonoscopy. PICO $(10 \mathrm{mg}$ of sodium picosulfate, $3.5 \mathrm{~g}$ of magnesium oxide, and $12 \mathrm{~g}$ of citric acid; Picolight, Pharmbio, Seoul, Korea) and $4 \mathrm{~L}$ of PEG (236 g of PEG, $22.97 \mathrm{~g}$ of potassium chloride, $5.86 \mathrm{~g}$ of sodium chloride, $6.74 \mathrm{~g}$ of sodium bicarbonate, and $22.74 \mathrm{~g}$ of anhydrous sodium sulfate; Dreampharma, Seoul, Korea) were used alone or in combination for this study. The following three regimens for bowel preparation were compared: 3 packets of PICO (group A), 2 packets of PICO with $1 \mathrm{~L}$ of PEG (group B), and 2 packets of PICO with $2 \mathrm{~L}$ of PEG (group C).

At $8 \mathrm{PM}$ on the day before the colonoscopy, the patients in group A were given the first packet of PICO mixed with $150 \mathrm{~mL}$ of water, followed by $1 \mathrm{~L}$ of water. On the examination day, they were given a second PICO packet at $5 \mathrm{AM}$ and a third PICO packet at 6 AM in the same manner. All three groups took 2 PICO packets in the same manner on the day of colonoscopy. The patients in group $\mathrm{B}$ were given $1 \mathrm{~L}$ of $\mathrm{PEG}$, and the patients in group $\mathrm{C}$ were given 2 $\mathrm{L}$ of PEG at $8 \mathrm{PM}$ before the day of colonoscopy, followed by 2 PICO packets on the day of colonoscopy.

\section{Questionnaire}

All the patients completed a questionnaire regarding sex, height and weight before the colonoscopy. We verified the understanding of all the patients regarding the bowel preparation method and the restricted-diet instructions. To evaluate the satisfaction and the preference of the patients, we used a "yes/no" questionnaire on whether the entire quantity of the regimen had been ingested. The taste of the product, the discomfort because of the preparation, and the residual portion of bowel preparation regimen were described.

Before the examination, the patients were asked about their satisfaction with the bowel preparation regimens and were asked to describe their past experience with colonoscopy. The patients were questioned regarding whether they wanted to receive the same bowel preparation protocol on their next examination.

The patients completed a questionnaire regarding the symptoms associated with the use of the bowel preparation regimen. They graded nausea, abdominal bloating, abdominal pain and sleep disturbance on a scale from 0 to 5 , with 0 being no symptoms and 5 indicating severe symptoms. We defined 'severe' or 'very severe' as a side effect. The colonoscopists scored the questionnaire regarding the overall degree of colon cleansing and the state of each segment of the colon after the examination.

\section{Colonoscopy}

Eight physicians who had performed over five hundred colonoscopies performed the procedure with the EC-3890Fi system (HOYA Co., Tokyo, Japan). For a colonoscopy with sedation, midazolam $(0.05 \mathrm{mg} / \mathrm{kg})$ and $3 \mathrm{mg}$ of propofol were injected intravenously, and apulse oximetry machine was attached to monitor the vital signs of the patient. We recorded thececal insertion time. The success of the procedure was determined by whether the colonoscope reached the cecum.

The Aronchick scoring scale and the Ottawa scale were used to evaluate the bowel's preparation state. Based on the Aronchick scale, the bowel preparation state was monitored as follows: "excellent" ( $>95 \%$ of the mucosa was observed, the stool was mostly liquid, and minimal suctioning was needed for adequate visualization), "good" ( $>90 \%$ of the mucosa wasobserved, the stool was mostly liquid, and significant suctioning was needed for adequate visualization), "inadequate" ( $<90 \%$ of the mucosa was observed, and the stool was a solid or semisolid mixture that could not be suctioned or washed).

The efficacy was assessed in the right colon, midcolon, and rectosigmoid segments of the colon by using the Ottawa scale. The bowel preparation state of the colon segments was reported by the colonoscopists who assigned a score based on a scale as follows: excellent, 0 ; good, 1 ; fair, 2 ; poor, 3 ; or inadequate, 4 . The colonoscopists rated the overall fluid amount on a 3 -point scale ( 0 , mild; 1 , moderate; 2 , severe). This remnant fluid assessment score was added to the scores obtained for each colon segment to derive a cumulative Ottawa scale score [5]. Before this study, the participating colonoscopists together had evaluated the bowel preparation states of thirty patients so as to reduce the differences between the colonoscopists and to increase the accuracy of the Ottawa measurement.

\section{Statistical analysis}

The patients' satisfaction, compliance, and bowel preparation state were assessed by using a chi-squared test, and the mean 
value and the standard deviation (SD) data were presented by using a one-way analysis of variance test. The statistical analysis was performed using IBM SPSS Statistics ver. 20.0 (IBM Co., Armonk, NY, USA). A calculated $\mathrm{P}<0.05$ was considered indicative of statistical significance (two-sided probability) and was provided as the mean \pm SD.

\section{RESULTS}

\section{Patient characteristics}

A total of 892 patients participated in the study and were divided into the following three groups: 282 patients were in group A, 303 patients were in group $\mathrm{B}$, and 307 patients were in group $\mathrm{C}$. The average age in the three groups was 52 years ( $20-70$ years). The average age was $51.7 \pm 9.6$ years in group A, $52.6 \pm 9.7$ years in group $B$, and $52.7 \pm 9.0$ years in group C. There was no significant difference in age, sex, height, weight, body mass index, and experience of colonoscopy in all the groups. The history of laxative use for constipation was not different among the three groups (Table 1).

\section{Pretreated colorectal and colonoscopy results}

The dietary restriction before colonoscopy of the three groups was not significantly different. The rate of the answer, "well understood", regarding the bowel preparation drug before colonoscopy was $95.7 \%$ (group A), $95.7 \%$ (group B), and $94.5 \%$ (group $\mathrm{C}$ ), showing no significant difference among the three groups. The completion rate of the bowel preparation was $95 \%$ in group A, $96 \%$ in group B, and $92 \%$ in group C. There was no significant difference among the three groups. The success of the colonoscopy and the cecal insertion time were not different significantly among the groups (Table 2).

\section{Satisfaction and preference}

We classified the patient satisfaction when the patients answered

Table 1. Patients' characteristics

\begin{tabular}{lcccc}
\hline Characteristic & $\begin{array}{c}\text { Group A } \\
(\mathrm{n}=282)\end{array}$ & $\begin{array}{c}\text { Group B } \\
(\mathrm{n}=303)\end{array}$ & $\begin{array}{c}\text { Group C } \\
(\mathrm{n}=307)\end{array}$ & P-value \\
\hline Male sex & $154(54.6)$ & $157(51.8)$ & $173(56.4)$ & NS \\
Age $(\mathrm{yr})$ & $51.7 \pm 9.6$ & $52.6 \pm 9.7$ & $52.7 \pm 9.0$ & NS \\
Height $(\mathrm{cm})$ & $166.0 \pm 7.2$ & $165.1 \pm 9.4$ & $165.0 \pm 7.9$ & NS \\
$\begin{array}{l}\text { Body weight }(\mathrm{kg}) \\
\begin{array}{l}\text { Body mass index } \\
\left(\mathrm{kg} / \mathrm{m}^{2}\right)\end{array}\end{array}$ & $25.4 \pm 7.9 \pm 9.5$ & $63.1 \pm 11.8$ & $63.5 \pm 9.7$ & NS \\
Pcol & $129(45.7)$ & $133(43.9)$ & $150(48.9)$ & NS \\
Lax & $6(2.1)$ & $8(2.6)$ & $8(2.6)$ & NS \\
\hline
\end{tabular}

Values are presented as number (\%) or mean \pm standard deviation.

Group A, 3 packets of sodium picosulfate/magnesium citrate; group B, 2 packets of sodium picosulfate/magnesium citrate $+1 \mathrm{~L}$ of polyethylene glycol; group $\mathrm{C}, 2$ packets of sodium picosulfate/magnesium citrate $+2 \mathrm{~L}$ of polyethylene glycol; $\mathrm{Pcol}$, history of previous colonoscopy; Lax, history of laxative drug; NS, no specific. 'very good' or 'good' for the assessment of the bowel preparation protocol. The satisfaction rate of the bowel preparation protocol was $72.0 \%$ (203 patients) in group A, 64.0\% (196 patients) in group $\mathrm{B}$, and $45.9 \%$ (141 patients) in group C. Groups A and B were statistically higher than group $\mathrm{C}$ in satisfaction (Table 3). Two hundred seven patients (73.4\%) in group A, 147 patients (48.7\%) in group $\mathrm{B}$, and 93 patients (30.3\%) in group $\mathrm{C}$ answered that there was no difficulty in drinking the bowel preparation regimens in the taste evaluation. Compared with the 3 packets of PICO, the PEG groups (groups B and C) showed a significant difference, which indicated difficulty in drinking the prescribed preparation $(\mathrm{P}<0.01)$ (Table 3).

The patients were asked about their willingness to select the same colon preparation regimen for a colonoscopy in the future. Of the answers, $89 \%$ (251 patients) in group A were positive, 79.1\% (239 patients) in group B were positive, and $72.3 \%$ (222 patients) in group $\mathrm{C}$ were positive. Group $\mathrm{C}$ indicated the lowest preference compared to the other groups $(\mathrm{P}<0.01)$ (Table 3$)$. Side effects, such as nausea and abdominal bloating, were noted by patients in group $\mathrm{C}$ more frequently than they were by patients in groups $\mathrm{A}$ and $\mathrm{B}(\mathrm{P}<0.01)$ (Table 4). No differences were noted in lack of sleep and abdominal pain among the three groups (Table 4 ).

Table 2. Bowel preparation and procedure results

\begin{tabular}{lcccc}
\hline Variable & $\begin{array}{c}\text { Group A } \\
(\mathrm{n}=282)\end{array}$ & $\begin{array}{c}\text { Group B } \\
(\mathrm{n}=303)\end{array}$ & $\begin{array}{c}\text { Group C } \\
(\mathrm{n}=307)\end{array}$ & P-value \\
\hline Diet & $270(95.7)$ & $291(96.0)$ & $292(95.1)$ & NS \\
Understanding & $270(95.7)$ & $290(95.7)$ & $290(94.5)$ & NS \\
$\begin{array}{l}\text { Fulfillment of procedure } \\
\quad \text { (well performed) }\end{array}$ & $266(94.3)$ & $290(95.7)$ & $282(91.9)$ & NS \\
$\begin{array}{l}\text { Success rate } \\
\text { Insertion time }\end{array}$ & $273(96.8)$ & $287(94.7)$ & $300(97.7)$ & NS \\
\hline
\end{tabular}

Values are presented as number (\%) or mean \pm standard deviation.

Group A, 3 packets of sodium picosulfate/magnesium citrate; group B, 2 packets of sodium picosulfate/magnesium citrate $+1 \mathrm{~L}$ of polyethylene glycol; group $\mathrm{C}, 2$ packets of sodium picosulfate/magnesium citrate $+2 \mathrm{~L}$ of polyethylene glycol; NS, no specific.

Table 3. Satisfaction with and preference for the preparation method

\begin{tabular}{lcccc}
\hline Variable & $\begin{array}{c}\text { Group A } \\
(\mathrm{n}=282)\end{array}$ & $\begin{array}{c}\text { Group B } \\
(\mathrm{n}=303)\end{array}$ & $\begin{array}{c}\text { Group C } \\
(\mathrm{n}=307)\end{array}$ & P-value \\
\hline Satisfaction & $203(72.0)^{\star}$ & $196(64.7)^{\star}$ & $141(45.9)$ & $<0.01$ \\
Taste & $207(73.4)^{\star}$ & $147(48.5)$ & $93(30.7)$ & $<0.01$ \\
$\begin{array}{l}\text { Preference for } \\
\text { preparation method }\end{array}$ & $251(89.0)^{\star}$ & $239(78.9)^{\star}$ & $222(72.3)$ & $<0.01$ \\
\hline
\end{tabular}

Values are presented as number (\%).

Group A, 3 packets of sodium picosulfate/magnesium citrate; group B, 2 packets of sodium picosulfate/magnesium citrate $+1 \mathrm{~L}$ of polyethylene glycol; group $\mathrm{C}, 2$ packets of sodium picosulfate/magnesium citrate $+2 \mathrm{~L}$ of polyethylene glycol. ${ }^{*}$ Statistical significance compare with group C. 
Table 4. Patients' tolerance responses

\begin{tabular}{lcccc}
\hline Variable & $\begin{array}{c}\text { Group A } \\
(\mathrm{n}=282)\end{array}$ & $\begin{array}{c}\text { Group B } \\
(\mathrm{n}=303)\end{array}$ & $\begin{array}{c}\text { Group C } \\
(\mathrm{n}=307)\end{array}$ & P-value \\
\hline Nausea & $65(23.0)$ & $104(34.3)$ & $117(38.1)$ & $<0.01$ \\
Fullness & $94(33.3)$ & $99(32.7)$ & $178(58.7)$ & $<0.01$ \\
Sleeplessness & $89(31.6)$ & $73(24.1)$ & $83(27.0)$ & NS \\
Abdominal cramp & $27(9.6)$ & $27(8.9)$ & $42(13.7)$ & NS \\
\hline
\end{tabular}

Values are presented as number (\%).

Group A, 3 packets of sodium picosulfate/magnesium citrate; group B, 2 packets of sodium picosulfate/magnesium citrate $+1 \mathrm{~L}$ of polyethylene glycol; group $\mathrm{C}, 2$ packets of sodium picosulfate/magnesium citrate $+2 \mathrm{~L}$ of polyethylene glycol; NS, no specific.

Table 5. Quality of bowel preparation (Aronchick scoring scale)

\begin{tabular}{lcccc}
\hline Variable & $\begin{array}{c}\text { Group A } \\
(\mathrm{n}=275)\end{array}$ & $\begin{array}{c}\text { Group B } \\
(\mathrm{n}=297)\end{array}$ & $\begin{array}{c}\text { Group C } \\
(\mathrm{n}=302)\end{array}$ & P-value \\
\hline Excellent & $67(24.4)$ & $54(18.2)$ & $76(25.2)$ & $<0.01$ \\
Good & $120(43.6)$ & $160(53.9)$ & $172(57.0)$ & \\
Fair & $69(25.1)$ & $60(20.2)$ & $41(13.6)$ & \\
Poor & $18(6.5)$ & $22(7.4)$ & $12(4.0)$ & \\
Inadequate & $1(0.4)$ & $1(0.3)$ & $1(0.3)$ & \\
\hline
\end{tabular}

Values are presented as number (\%).

Group A, 3 packets of sodium picosulfate/magnesium citrate; group B, 2 packets of sodium picosulfate/magnesium citrate $+1 \mathrm{~L}$ of polyethylene glycol; group $\mathrm{C}, 2$ packets of sodium picosulfate/magnesium citrate $+2 \mathrm{~L}$ of polyethylene glycol.

\section{Bowel cleansing efficacy}

Each group included one patient who could not complete the total bowel preparation process because of the taste. As to the number of bowel preparations rated as 'excellent' and 'good', the numbers in groups $\mathrm{A}$ and $\mathrm{C}$ were significantly higher than the number in group $B(P<0.01)$ (Table 5). In the bowel preparation using the Ottawa scale assessment, group $C$ showed better cleansing in the right colon than groups $\mathrm{A}$ and $\mathrm{B}$. In the transverse colon segment, rectum, and sigmoid colon, group $C$ showed better cleansing than did group $B(P<0.05)$. No difference was between groups $A$ and $\mathrm{C}$ (Table 6). The amount of colon residual fluid in the group $\mathrm{C}$ patients was less than it was in the group $\mathrm{B}$ patients $(\mathrm{P}<0.01)$ (Table 6). On the Ottawa scale, group $\mathrm{C}$ showed better results than group $\mathrm{B}(\mathrm{P}<0.01)$. No significant difference was noted between groups $\mathrm{A}$ and $\mathrm{C}$.

\section{DISCUSSION}

Colonoscopy is essential for the accurate diagnosis and treatment of colorectal lesions. For efficient colonoscopy, adequate bowel preparation is crucial, and less residual liquid and solid stool in the mucosa is important $[6,7]$. Research on various bowel preparation regimens has been conducted. PEG, a 4-L dosage, is the most efficient and safe regimen. PEG is an isotonic electrolyte solution that was introduced in the 1980s by Davis. Patients do not
Table 6. Comparison of the Ottawa bowel preparation quality scale scores between the groups

\begin{tabular}{lcccc}
\hline Colonic segment & $\begin{array}{c}\text { Group A } \\
(\mathrm{n}=282)\end{array}$ & $\begin{array}{c}\text { Group B } \\
(\mathrm{n}=303)\end{array}$ & $\begin{array}{c}\text { Group C } \\
(\mathrm{n}=307)\end{array}$ & P-value \\
\hline Right colon & $1.34 \pm 1.022$ & $1.45 \pm 0.97$ & $1.11 \pm 0.97^{\mathrm{a}, \mathrm{b}}$ & 0.02 \\
Mid colon & $1.13 \pm 0.90$ & $1.28 \pm 0.89$ & $1.11 \pm 0.98^{\mathrm{a}}$ & 0.05 \\
Rectosigmoid colon & $1.13 \pm 0.95$ & $1.22 \pm 0.91$ & $0.97 \pm 0.87^{\mathrm{a}}$ & 0.03 \\
Fluid volume (mL) & $0.53 \pm 0.63$ & $0.59 \pm 0.66$ & $0.37 \pm 0.56^{\mathrm{a}, \mathrm{b}}$ & $<0.01$ \\
Ottawa scale & $4.13 \pm 2.95$ & $4.44 \pm 2.79$ & $3.50 \pm 2.84^{\mathrm{a}}$ & $<0.01$ \\
\hline
\end{tabular}

Values are presented as mean \pm standard deviation.

Group A, 3 packets of sodium picosulfate/magnesium citrate; group B, 2 packets of sodium picosulfate/magnesium citrate $+1 \mathrm{~L}$ of polyethylene glycol; group $\mathrm{C}, 2$ packets of sodium picosulfate/magnesium citrate $+2 \mathrm{~L}$ of polyethylene glycol; Right colon, cecum and ascending colon; Mid colon, transverse and descending colon.

${ }^{\mathrm{a} A}$ significant difference exists between groups $\mathrm{C}$ and $\mathrm{B}$. ${ }^{\mathrm{b}} \mathrm{A}$ significant difference exists between groups $C$ and $A$.

need a restricted meal before a colonoscopy, and it is taken for a short time; thus, compared to the other colon preparation drugs, PEG is economical, convenient, and relatively safe for patients, including those with circulatory and kidney disease. Patients should take a large amount of the 4-L PEG solution within 3-4 hours, and the taste is unpleasant. NaP might cause intravascular high osmotic pressure, resulting in the loss of body water, transient hyperphosphatemia and hypocalcemia [8], as well as seizures and decreased consciousness accompanied by complications such as hyponatremia [9]. In November 2009, the FDA prohibited the use of $\mathrm{NaP}$ as a colon preparation drug.

Combinations of various bowel preparation solutions have been attempted because of the disadvantages of PEG and the prohibition of NaP usage. Recently, PICO has been used for colonoscopy or barium-enema cleansing for adults and children because it has a low volume and a better taste than PEG while having a similar efficacy in colon cleansing [2]. PICO is dispensed in powder form ( $0.01 \mathrm{~g}$ of sodium picosulfate, $3.5 \mathrm{~g}$ of magnesium oxide, and 12.0 $\mathrm{g}$ of citric acid per packet), with the magnesium oxide and citric acid components forming magnesium citrate when the powder is dissolved in water. Sodium picosulfate/magnesium citrate acts locally in the colon as a stimulant laxative by increasing the frequency and the force of peristalsis and promoting electrolyte and water retention in the colon (the sodium picosulfate component) and as an osmotic laxative by retaining fluids in the colon (the magnesium citrate component) to clear the colon and the rectum of their fecal contents. Two packets of sodium picosulfate/magnesium citrate and $236 \mathrm{~g}$ of PEG orally show similar bowel preparation efficacies in adult patients [2]. Previous studies on PICO [4, 10] have used two packets in split doses; a 3-packet dose of PICO has not been studied sufficiently $[11,12]$.

Because of the difficulty drinking $4 \mathrm{~L}$ of PEG, $5 \%-15 \%$ of patients are reported as having failed in bowel preparation [13]. In the aspect of taste, group A patients were more positive answer 
than groups B and C patients. Each PICO packet contains $12 \mathrm{~g}$ of orange-flavored citric acid, which provides a better taste compared to the other regimens. The patients were asked, "Are you willing to take the same regimen for the next colonoscopy?" The patients in group A answered much more positively than did those in groups $\mathrm{B}$ and $\mathrm{C}$ because the regimen used for group $\mathrm{A}$ required relatively small volumes (each packet was mixed with $150 \mathrm{~mL}$ of water) and had a more pleasant taste than the regimens used for groups B and C [3].

PICO has been reported to induce abdominal pain, nausea, daily headaches, and a mild degree of sleep disturbance [2]. In one study, 3.0\% of the patients reported nausea with a PICO dosage of 2 packets [14], and compared to the 3-packet split-dose PICO group and $90 \mathrm{~mL}$ of $\mathrm{NaP}$ group, the PICO group was less likely to develop side effects, such as dizziness, nausea, vomiting, and abdominal pain [11]. In this study, sleep disturbance and abdominal pain were similar in the three groups. The patients in groups A and B were less likely than those in group C to report nausea and abdominal bloating. The findings of less nausea in the PICO regimen compared with that in the PEG regimen is consistent with previous studies [2]. The rates of nausea, abdominal bloating, and other side effect were high in this study because most patients checked 'yes' to all symptoms even though they were minimal.

Flemming et al. [15] reported that the efficacy of bowel cleansing varied depending on the administration time of the bowel preparation regimen. The PICO split-dose regimen reported good efficacy in the case of a morning colonoscopy; however, if a colonoscopy was scheduled in the afternoon, PICO administration four hours prior to colonoscopy showed good results. In this study, we administered a split-dose PICO regimen, including an additional administration 3-4 hours prior to the colonoscopy. Love et al. [3] reported that in $93 \%-96 \%$ of patients, cleansing was adequate or excellent with Sodium picosulfate magnesium citrate 2 PICO packets. The result of our study was nearly identical. The overall colon cleansing efficacy of groups $\mathrm{A}$ and $\mathrm{C}$ were better than that of group B. Group C showed better cleansing and less liquid sequestration in the right colon than did group $\mathrm{A}$. The addition of $10 \mathrm{mg}$ of bisacodyl to the regimen has been reported in the literature to improve the cleansing effect of the entire colon [10]. Further study is warranted to investigate this combination.

Side effects such as weight loss, dehydration, increased hemoglobin [2], hyponatremia, and syncope after administration of 2 packets of PICO have been reported [16]. Orthostatic hypotension has been noted in elderly patients and patients with risk factors [2]. Although not clinically significant, the possibility of hypokalemia, hypocalcemia, and hypermagnesemia might be high. Generally, calcium and potassium levels returned to normal within 24 hours after the PICO regimen had been completed [17].

In the literature, PICO has been introduced as an effective and safe bowel preparation regimen before colonoscopy for children, adolescents and elderly patients. PICO showed particularly high compliance in children $[18,19]$. Hypermagnesemia might develop from the use of four packets of PICO; however, no renal dysfunction or electrolyte imbalance has been observed [20].

Lawrance et al. [21] reported more mucosal inflammation with $\mathrm{NaP}$ and PICO than with PEG.

The limitation of this study is that the data are from a specific population, in that the ages of our patients varied from 20 to 70 years of age. In addition, no data were available regarding the analysis of fluids and electrolytes. However, the following conclusions can be drawn: group A showed a better result than groups B and $\mathrm{C}$ in the aspect of patient satisfaction and preference. In the aspect of cleansing quality, no difference was observed between groups A and C. Further study is necessary concerning the safety of using three packets of PICO for a bowel preparation regimen.

\section{CONFLICT OF INTEREST}

No potential conflict of interest relevant to this article was reported.

\section{REFERENCES}

1. Tan JJ, Tjandra JJ. Which is the optimal bowel preparation for colonoscopy: a meta-analysis. Colorectal Dis 2006;8:247-58.

2. Hoy SM, Scott LJ, Wagstaff AJ. Sodium picosulfate/magnesium citrate: a review of its use as a colorectal cleanser. Drugs 2009;69: 123-36.

3. Love J, Bernard EJ, Cockeram A, Cohen L, Fishman M, Gray J, et al. A multicentre, observational study of sodium picosulfate and magnesium citrate as a precolonoscopy bowel preparation. Can J Gastroenterol 2009;23:706-10.

4. Worthington J, Thyssen M, Chapman G, Chapman R, Geraint M. A randomised controlled trial of a new 2 litre polyethylene glycol solution versus sodium picosulphate + magnesium citrate solution for bowel cleansing prior to colonoscopy. Curr Med Res Opin 2008:24:481-8.

5. Rostom A, Jolicoeur E. Validation of a new scale for the assessment of bowel preparation quality. Gastrointest Endosc 2004;59: 482-6.

6. Sharma VK, Steinberg EN, Vasudeva R, Howden CW. Randomized, controlled study of pretreatment with magnesium citrate on the quality of colonoscopy preparation with polyethylene glycol electrolyte lavage solution. Gastrointest Endosc 1997;46:541-3.

7. Kim WH, Cho YJ, Park JY, Min PK, Kang JK, Park IS. Factors affecting insertion time and patient discomfort during colonoscopy. Gastrointest Endosc 2000;52:600-5.

8. Huynh T, Vanner S, Paterson W. Safety profile of 5-h oral sodium phosphate regimen for colonoscopy cleansing: lack of clinically significant hypocalcemia or hypovolemia. Am J Gastroenterol 1995;90:104-7.

9. Baik SJ, Shim KN, Na YJ, Kang MJ, Jung JM, Jung SA, et al. Hyponatremia with seizure and mental change after oral sodium 
phosphate bowel preparation: report of two cases. Korean J Gastrointest Endosc 2008;37:55-60.

10. Hookey LC, Vanner SJ. Pico-salax plus two-day bisacodyl is superior to pico-salax alone or oral sodium phosphate for colon cleansing before colonoscopy. Am J Gastroenterol 2009;104:703-9.

11. Schmidt LM, Williams P, King D, Perera D. Picoprep-3 is a superior colonoscopy preparation to Fleet: a randomized, controlled trial comparing the two bowel preparations. Dis Colon Rectum 2004; 47:238-42.

12. Longcroft-Wheaton G, Bhandari P. Same-day bowel cleansing regimen is superior to a split-dose regimen over 2 days for afternoon colonoscopy: results from a large prospective series. J Clin Gastroenterol 2012;46:57-61.

13. DiPalma JA, Marshall JB. Comparison of a new sulfate-free polyethylene glycol electrolyte lavage solution versus a standard solution for colonoscopy cleansing. Gastrointest Endosc 1990;36:285-9.

14. Katz PO, Rex DK, Epstein M, Grandhi NK, Vanner S, Hookey LC, et al. A dual-action, low-volume bowel cleanser administered the day before colonoscopy: results from the SEE CLEAR II study. Am J Gastroenterol 2013;108:401-9.

15. Flemming JA, Vanner SJ, Hookey LC. Split-dose picosulfate, magnesium oxide, and citric acid solution markedly enhances colon cleansing before colonoscopy: a randomized, controlled trial. Gastrointest Endosc 2012;75:537-44.
16. Dillon CE, Laher MS. The rapid development of hyponatraemia and seizures in an elderly patient following sodium picosulfate/ magnesium citrate (Picolax). Age Ageing 2009;38:487.

17. Rahman A, Vanner SJ, Baranchuk A, Hookey LC. Serial monitoring of the physiological effects of the standard Pico-Salax ${ }^{\circledast}$ regimen for colon cleansing in healthy volunteers. Can J Gastroenterol 2012;26:424-8.

18. Turner D, Levine A, Weiss B, Hirsh A, Shamir R, Shaoul R, et al. Evidence-based recommendations for bowel cleansing before colonoscopy in children: a report from a national working group. Endoscopy 2010;42:1063-70.

19. Turner D, Benchimol EI, Dunn H, Griffiths AM, Frost K, Scaini V, et al. Pico-Salax versus polyethylene glycol for bowel cleanout before colonoscopy in children: a randomized controlled trial. Endoscopy 2009;41:1038-45.

20. Mc Laughlin P, Eustace J, Mc Sweeney S, Mc Williams S, O’Regan $\mathrm{K}$, O'Connor $\mathrm{M}$, et al. Bowel preparation in CT colonography: electrolyte and renal function disturbances in the frail and elderly patient. Eur Radiol 2010;20:604-12.

21. Lawrance IC, Willert RP, Murray K. Bowel cleansing for colonoscopy: prospective randomized assessment of efficacy and of induced mucosal abnormality with three preparation agents. Endoscopy 2011;43:412-8. 